\title{
Actitud de los estudiantes universitarios frente a la educación virtual en tiempos de la pandemia de COVID-19
}

\author{
Edwin Gustavo Estrada Araoz ${ }^{1}$, Néstor Antonio Gallegos Ramos ${ }^{2}$, Helen Juddy Mamani Uchasara ${ }^{3}$, Karl Herbert Huaypar \\ Loayza $^{4}$ \\ 1, 2,3, 4 Universidad Nacional Amazónica de Madre de Dios. Facultad de Educación. Avenida Jorge Chávez 1160. Perú. \\ Autor para correspondência/Author for correspondence: edwin5721@outlook.com
}

RESUMEN. El objetivo de la presente investigación fue analizar la actitud de los estudiantes de la Universidad Nacional Amazónica de Madre de Dios frente a la educación virtual en tiempos de la pandemia de COVID-19. El enfoque de investigación fue cuantitativo, el diseño no experimental y el tipo de diseño transeccional descriptivo. La muestra estuvo conformada por 154 estudiantes de ambos sexos de la carrera profesional de educación y fue determinada mediante un muestreo probabilístico estratificado. El instrumento utilizado para la recolección de datos fue la Escala de Actitudes frente a la educación virtual, la cual cuenta con la confiabilidad y validez requerida. En base a los resultados se demostró que la mayoría de estudiantes presentan una actitud de indiferencia frente a la educación virtual y se hallaron diferencias estadísticamente significativas entre el sexo y la edad de los estudiantes.

Palabras clave: Actitudes, Educación Virtual, Educación Superior Universitaria, Educación, COVID-19. 


\title{
Attitude of university students towards virtual education during the COVID-19 pandemic
}

\begin{abstract}
The objective of this research was to analyze the attitude of the students of the National Amazonian University of Madre de Dios towards virtual education in times of the COVID-19 pandemic. The research focus was quantitative, the non-experimental design and the descriptive transitional type of design. The sample consisted of 154 students of both sexes from the professional education, career and was determined by means of a stratified probabilistic sampling. The instrument used for data collection was the Scale of Attitudes towards virtual education, which has the required reliability and validity. Based on the results, it was shown that the majority of students present an attitude of indifference towards virtual education and statistically significant differences were found between the sex and age of the students.
\end{abstract}

Keywords: Attitudes, Virtual Education, University Higher Education, Education, COVID-19. 


\section{Atitude de estudantes universitários em relação à educação virtual durante a pandemia do COVID-19}

RESUMO. O objetivo desta pesquisa foi analisar a atitude dos estudantes da Universidade Nacional da Amazônia de Madre de Dios em relação à educação virtual em tempos da pandemia do COVID-19. O foco da pesquisa foi quantitativo, com desenho não experimental e do tipo transicional descritivo. A amostra foi composta por 154 estudantes de ambos os sexos da carreira de educação profissional e foi determinada por meio de uma amostragem probabilística estratificada. $\mathrm{O}$ instrumento utilizado para a coleta de dados foi a Escala de Atitudes em relação à educação virtual, que possui a confiabilidade e validade necessárias. Com base nos resultados, demonstrou-se que a maioria dos estudantes apresenta uma atitude de indiferença em relação à educação virtual e foram encontradas diferenças estatisticamente significantes entre o sexo e a idade dos estudantes.

Palavras-chave: Atitudes, Educação Virtual, Ensino Superior Universitário, Educação, COVID-19. 


\section{Introducción}

El avance vertiginoso de las tecnologías de la información y la comunicación (TIC) ha producido grandes cambios positivos en los diferentes ámbitos de la vida, y como es de suponer, la educación no fue la excepción, siendo fortalecida por el surgimiento de la virtualización de la educación (Pulido, 2017; Moreira y Delgadillo, 2015). Por ello, la educación virtual ha ido creciendo durante los últimos años debido a que esta modalidad no tiene fronteras y permite que los estudiantes accedan a ellas, a diferencia de la modalidad presencial, de manera flexible (Hernández, Fernández y Pulido, 2018).

De la misma manera, también las propuestas de educación virtual buscan revolucionar la educación con la finalidad de influir favorablemente en el proceso de enseñanza - aprendizaje (Pando, 2018). Bajo esa premisa, existe una tendencia que apunta a que más personas estudien en entornos flexibles, en ese sentido, la educación virtual es una herramienta que se muestra cada vez más eficaz, para satisfacer esa tendencia y solucionar los problemas de acceso y calidad en la educación (Campillo, et al., 2013). Por ello, la educación virtual actualmente, "representa una alternativa ideal para un importante grupo de estudiantes ya que les permite compatibilizar su actividad laboral y familiar con su formación, al desarrollarla en su propio domicilio" (Durán, Estay y Álvarez, 2015, p. 78).

De acuerdo a Cruz y Rama (2016), la educación virtual es una modalidad que se desprende de la educación a distancia, en la que los aprendizajes son mediados por la tecnología, a través de herramientas digitales, espacios virtuales y plataformas, añadiéndose así a la educación superior las bondades del uso de las TIC. Por otro lado, Aguilar (2015) señala que la educación virtual hace referencia a la utilización de las tecnologías que se basan principalmente en el internet para brindar una amplia gama de soluciones y así desarrollar conocimientos y habilidades en los estudiantes.

La educación virtual es una modalidad educativa que optimiza y mejora la calidad de la enseñanza y del aprendizaje puesto que toma en cuenta su flexibilidad o disponibilidad, es decir, los estudiantes pueden organizar sus tiempos y espacios de estudio en función a su disponibilidad (Lara, 2002). Asimismo, Rivera, Viera y Pulgarón (2010) señalan que entre las principales bondades de esta modalidad está su dinamismo e interactividad pues los docentes y los estudiantes mantienen una comunicación constante a pesar de que se encuentran 
separados físicamente. En ese sentido, la tecnología tiene un importante rol puesto que mediante ella se pueden realizar videoconferencias, enviar mensajes $\mathrm{y}$ correos electrónicos, compartir información y evaluar los aprendizajes de los estudiantes.

Al respecto, Patiño y Palomino (2004) mencionan que la educación virtual pueda darse a través de una interacción en tiempo real, la cual es denominada síncrona, cuyas principales herramientas son la videoconferencia y los chats. Por otro lado, si la interacción entre el docente y los estudiantes se da de manera diferida, estaríamos refiriéndonos a la interacción asíncrona, teniendo como principales herramientas al foro, las tareas, los correos electrónicos y los wikis (Herrera et al., 2019).

Un aspecto importante para el éxito de la educación virtual es el papel de los estudiantes, quienes tienen que adaptarse a esta modalidad de estudios. Por ello, deberán estar motivados, ser responsables, autónomos y eficientes para organizar y gestionar su tiempo, desarrollar sus actividades de manera adecuada y entregarlas oportunamente. Bajo esa perspectiva, la decisión de afrontar y terminar satisfactoriamente un curso $\mathrm{o}$ programa educativo virtual es determinada por varios factores, sin embargo, uno de los principales es la actitud del estudiante (Hernández, Fernández y Pulido, 2018).

Según Ajzen (1991) la actitud es un factor que tiene influencia en las decisiones que toman las personas para actuar de determinada manera. En ese sentido, la actitud está relacionada al sentimiento favorable o desfavorable de los estudiantes para llevar a cabo una determinada acción. Para De las Salas, Perozo y Lugo (2015) la actitud está compuesta por 3 componentes: el componente cognitivo, orientado a los sentimientos que tienen los estudiantes, en este caso, sobre el funcionamiento $\mathrm{y}$ dominio tecnológico del estudiante para educarse virtualmente; el componente afectivo, referido al sentimiento de agrado o desagrado de la educación virtual; y el componente conductual, el cual está asociado a la tendencia que tienen los estudiantes para actuar, es decir, para ser partícipes de la educación virtual e involucrarse en el desarrollo de las actividades que en ella se programen.

Actualmente, la coyuntura social ha promovido la masificación de esta modalidad de estudios a nivel global. Como sabemos, el desarrollo de las actividades académicas en las instituciones educativas básicas y superiores a nivel mundial se ha visto interrumpido por la expansión de la pandemia COVID-19, la 
cual obligó a gran parte de la población a mantenerse en aislamiento social obligatorio y por ende, a cambiar la modalidad de aprendizaje, pasando de ser presencial o semipresencial a ser estrictamente virtual (Instituto de Investigaciones sobre la Universidad y la Educación, 2020; Moreno, 2020).

Bajo esa perspectiva, la modalidad virtual cambió el rol de los docentes y estudiantes así como el escenario donde se desarrollaba el proceso de enseñanza aprendizaje (Gómez y Macedo, 2011). El desafío ha sido entonces integrar prácticas educativas poco habituales e innovadoras, adaptándolas de manera sincrónica o asincrónica según corresponda en un contexto novedoso y poco manejable (Núñez, 2020).

En el caso de la educación superior universitaria peruana, la virtualización de la educación supuso un gran reto a las universidades y a su vez trajo consigo muchas dificultades debido especialmente a que muchas de ellas no contaban con aulas virtuales. Del mismo modo, los docentes y estudiantes tenían poca familiarización con los recursos y herramientas que tenían dichas aulas y sobre todo, por la brecha tecnológica que existe, la cual impide que todos los estudiantes puedan conectarse al internet y desarrollar las actividades académicas, tanto sincrónicas como asincrónicas.

De acuerdo a los datos proporcionados por el Instituto Nacional de Estadística e Informática (2018), solo el $28,04 \%$ de la población peruana censada en el año 2017 tenía acceso a internet y apenas el 33,74\% tenía una computadora, laptop o tablet. Ello implica que existe un gran desafío en cuanto a la virtualización de la educación, no solo para las universidades, sino para el Estado, el cual deberá establecer políticas que aseguren la conectividad al internet en todo el territorio nacional.

A pesar de las dificultades expuestas muchas de las universidades empezaron sus clases entre los meses de abril y mayo. Sin embargo, otra situación desafiante con la que se encontró fue, tal como se mencionó, la familiarización y el correcto uso de las aulas virtuales por parte de los docentes, lo que pone en evidencia a su vez las escasas estrategias didácticas que ellos poseen para desarrollar las actividades académicas en la modalidad virtual y estaría generando descontento e insatisfacción en el estudiantado frente a dicha modalidad debido a la forma cómo los docentes gestionan los aprendizajes.

En el caso de la Universidad Nacional Amazónica de Madre de Dios, desde el año 2019 cuenta con un aula 
virtual denominado Laurassia, el cual sirve como soporte fundamental en el proceso de enseñanza y contiene herramientas $y$ recursos digitales tales como el chat, calendario, tareas, exámenes, foros, lecturas, calificaciones, grupos, videoconferencia y wikis. Sin embargo, se puede percibir que los estudiantes han empezado a tener actitudes desfavorables frente a la educación virtual debido a que tienen una sobrecarga de trabajos y a la prolongada exposición a las laptops, computadoras, tablets y celulares, lo que también les provocaría cansancio visual, fatiga y estrés.

Frente a esta situación se planteó como pregunta de investigación ¿Cuál es la actitud de los estudiantes de la Universidad Nacional Amazónica de Madre de Dios frente a la educación virtual en tiempos de la pandemia de COVID-19?

Entre los principales antecedentes se encuentran Hernández, Fernández y Pulido (2018) quienes investigaron la actitud frente a la educación en línea en estudiantes de pregrado y posgrado en una universidad de Guatemala, concluyendo que ellos tenían una actitud positiva frente a la modalidad virtual. Del mismo modo, Pulido (2017) investigó sobre la actitud hacia la educación virtual en estudiantes de posgrado de una universidad venezolana, llegando a concluir que la mayoría de estudiantes tiene una actitud favorable ante la educación virtual, sin embargo, algunos de ellos presentaban dificultades para usar las herramientas virtuales y demostraban incertidumbre en cuanto a la intención de estudiar en el futuro bajo la mencionada modalidad. También Chávez y Rodríguez (2018) investigaron sobre actitud de los estudiantes universitarios mexicanos hacia la educación en línea, concluyendo que existe una actitud poco favorable hacia dicha modalidad. Por último, Maldonado y Vega (2015) estudiaron sobre la actitud de los estudiantes universitarios frente a la plataforma Moodle, llegando a concluir que, de manera general, tenían una actitud favorable hacia el uso de la plataforma de teleformación Moodle.

Con relación a la relevancia de la presente investigación, los hallazgos que fueron obtenidos serán de mucha importancia para la alta dirección de la universidad ya que podrán tomar acciones correctivas como capacitaciones y talleres que reorienten el proceso de enseñanza y fomenten la utilización adecuada de las herramientas y recursos del aula virtual. Asimismo, servirá como referencia a futuros estudios, ya que esta investigación es novedosa y a su vez aborda un problema coyuntural, no solo en la universidad, sino en la región de Madre de Dios, el Perú y el mundo. 
Teniendo en cuenta lo manifestado, el objetivo fue analizar la actitud de los estudiantes de la Universidad Nacional Amazónica de Madre de Dios frente a la educación virtual en tiempos de la pandemia de COVID-19.

Finalmente, es necesario señalar que esta investigación está estructurada por una introducción, metodología, resultados, discusión, consideraciones finales $\mathrm{y}$ referencias que permitirán una mejor comprensión de la misma.

\section{Metodología}

El enfoque de investigación fue cuantitativo ya que se procedió a recoger datos a partir de los instrumentos aplicados para realizar el análisis estadístico y así obtener información sistematizada (Sánchez, Reyes y Mejía, 2018). Asimismo, el diseño fue no experimental debido a que la variable no fue manipulada deliberadamente, sino fue observada tal como se dio en su entorno. Por otro lado, el tipo fue descriptivo transeccional ya que se analizaron las propiedades y características de la variable actitud ante la educación virtual y la recolección de datos se dio en un determinado momento (Hernández et al., 2014).

\section{Población y muestra}

La población fue conformada por 510 estudiantes matriculados y la muestra por 154 estudiantes de la carrera profesional de Educación, en sus tres especialidades: Inicial y Especial, Primaria e Informática y Matemática y Computación, matriculados en el ciclo 2020-I. Dicha cantidad fue determinada a través de un muestreo probabilístico estratificado. En la tabla 1 se detalla su distribución.

Tabla 1 - Distribución de la población y muestra.

\begin{tabular}{|c|c|c|c|}
\hline Especialidade & & Población & Muestra \\
\hline $\begin{array}{l}\text { Inicial } \\
\text { Especial }\end{array}$ & $\mathrm{y}$ & 250 & 75 \\
\hline $\begin{array}{l}\text { Primaria } \\
\text { Informática }\end{array}$ & e & 179 & 54 \\
\hline $\begin{array}{l}\text { Matemática } \\
\text { Computación }\end{array}$ & $\mathrm{y}$ & 81 & 25 \\
\hline Total & & 510 & 154 \\
\hline
\end{tabular}

Fuente: Dirección Universitaria de Asuntos Académicos.

Los estudiantes que fueron parte de la muestra se caracterizaron por pertenecer a niveles socioeconómicos medios y bajos. Es así que algunos de ellos reciben subvenciones económicas por parte del Estado para continuar sus estudios. Por otro lado, eran muy pocos los estudiantes que contaban con la conectividad adecuada y los planes de internet suficientes para afrontar sus estudios virtuales con normalidad. Finalmente, es menester precisar que un aspecto favorable fue que la mayoría tenía habilidades tecnológicas 
necesarias para desenvolverse en entornos virtuales.

\section{Técnicas e instrumentos}

La técnica utilizada para realizar la recolección de datos fue la encuesta y el instrumento fue la Escala de actitudes frente a la educación virtual, la cual fue elaborada por Mehra y Omidian (2012), sin embargo, debido a que la versión original estaba en inglés, fue necesario traducirla al español y adaptarla. Está conformada por 40 ítems de tipo Likert (muy de acuerdo, de acuerdo, parcialmente de acuerdo, en desacuerdo, muy en desacuerdo) los cuales evalúan 5 dimensiones: Percepción de utilidad (ítems del 1 al 12), intención de adoptar la educación virtual (ítems del 13 al 20), facilidad de uso, (ítems del 21 al 25), soporte pedagógico y técnico (ítems del 26 al 34) y estresores de la educación virtual (ítems del 35 al 40).

Sus propiedades métricas fueron determinadas mediante la validez y confiabilidad. La validación de contenido se realizó a través de la técnica de juicio de expertos, para lo cual se recurrió a 3 especialistas, quienes evaluaron la claridad de las preguntas, la congruencia, la coherencia y la pertinencia. El coeficiente $\mathrm{V}$ de Aiken fue de 0,808 lo que indica que la escala tiene buena validez. Respecto a la confiabilidad, fue hallada mediante una prueba piloto, obteniéndose un coeficiente Alfa de Cronbach de 0,891 lo que quiere decir que la escala es altamente confiable.

\section{Procedimientos}

Para la recolección de datos se contactó a los estudiantes para comunicarles el propósito de la investigación. Posteriormente, una vez que brindaron su consentimiento, cada estudiante recibió el enlace para acceder y desarrollar el cuestionario mediante el formulario de Google. Dicho proceso fue realizado en el mes de junio y el acceso a la encuesta fue cerrado al recibir 154 respuestas. Finalmente, después de obtener toda la información se consolidó en una base de datos donde se realizó el proceso de calificación de acuerdo a las normas de corrección y el análisis descriptivo e inferencial utilizando el software SPSS versión 22.

\section{Resultados}

Luego de aplicar el instrumento se procedió a estructurar la base de datos y así valorar las puntuaciones para luego elaborar las tablas, las cuales se exponen a continuación. 
Tabla 2 - Resultado descriptivo de la variable y sus dimensiones.

\begin{tabular}{lcccccc}
\hline \multicolumn{1}{c}{ Variable y dimensiones } & \multirow{2}{*}{$\mathrm{N}$} & \multirow{2}{*}{ Media } & \multirow{2}{*}{$\mathrm{DE}$} & \multicolumn{2}{c}{ IC 95\% } & \multirow{2}{*}{ Categoría } \\
\cline { 5 - 6 } & & & & Inferior & Superior & \\
Percepción de utilidad & 154 & 33,02 & 8,961 & 31,59 & 34,45 & Indiferencia \\
Intención de adoptar la educación virtual & 154 & 24,53 & 5,158 & 23,70 & 25,35 & Indiferencia \\
Facilidad de uso & 154 & 14,01 & 3,918 & 13,38 & 14,63 & Indiferencia \\
Soporte pedagógico y técnico & 154 & 22,05 & 6,184 & 21,06 & 23,03 & Rechazo \\
Estresores de la educación virtual & 154 & 23,45 & 6,914 & 22,83 & 24,08 & Alto \\
Actitud frente a la educación virtual & 154 & 107,05 & 22,048 & 103,54 & 110,56 & Indiferencia \\
\hline
\end{tabular}

Fuente: Base de datos.

En la tabla 2 se consolidaron los resultados descriptivos de la variable actitud frente a la educación virtual y sus respectivas dimensiones. En ese sentido, se puede observar que las medias de las dimensiones percepción de la utilidad, intención de adoptar la educación virtual y facilidad de uso fueron de 33,02; 24,53 y 14,01 respectivamente, lo cual permitió su categorización en el nivel de "indiferencia". Los resultados expuestos indican que los estudiantes consideran que la educación virtual es una alternativa que podría mejorar los procesos de enseñanza aprendizaje así como su rendimiento, sin embargo, sienten que no están preparados actualmente para estudiar bajo esta modalidad ya que se sienten poco motivados y atraídos para realizar las actividades a través de la plataforma virtual, asimismo, la interacción con los docentes y sus compañeros no es muy fluida y afirman que es mejor y más efectivo trabajar de manera presencial por su practicidad y dominio para desempeñarse en ella.
En cuanto a la dimensión soporte pedagógico y técnico, la media fue de 22,05 lo que indica que existe una actitud de rechazo. Esto significa que ellos no recibieron una orientación adecuada para desenvolverse en el aula virtual, asimismo, frente a las dificultades técnicas que encontraron en el proceso de desarrollo de sus actividades académicas, no fueron apoyados oportunamente por el personal competente. Por otra parte, muchos estudiantes además indicaron que no cuentan con los equipos necesarios para poder acceder a ella ni con una adecuada conectividad al internet para poder interactuar sincrónica o asincrónicamente con sus docentes así como compañeros y desarrollar las actividades propuestas.

Con relación a la dimensión estresores de la educación virtual, su media fue de 23,45; por lo que fue categorizada como alto. Esto significa que la educación virtual les está generando mayor ansiedad y estrés a los estudiantes en comparación a la educación presencial debido a los problemas de conectividad a internet y en otros casos a las limitaciones económicas 
que no les permiten poder contar con los recursos tecnológicos propicios para interactuar en dicha modalidad (laptops, tablets o celulares). Además, según la percepción de los estudiantes, otro factor asociado al estrés y ansiedad es originado debido a que muchos docentes no están capacitados para enseñar bajo modalidad virtual, en consecuencia, no aplican buenas estrategias de enseñanza y tienden a dejar muchas tareas.

Finalmente, respecto a la variable actitud frente a la educación virtual, su media fue de 107,05 lo cual significa que los estudiantes se caracterizan por tener una actitud de indiferencia, es decir, a pesar que consideran que la educación puede ser útil, las situaciones que están experimentando generan insatisfacción y poca disposición para afrontarla. En ese sentido, de mantenerse las condiciones expresadas, aumentaría la posibilidad de que la tasa de deserción escolar se incremente.

Tabla 3 - Actitud de los estudiantes frente a la educación virtual según especialidad.

\begin{tabular}{lccccc}
\hline \multirow{2}{*}{ Especialidad } & \multicolumn{4}{c}{ Actitud frente a la educación virtual } & Total \\
\cline { 2 - 5 } & $\begin{array}{c}\text { Mucho } \\
\text { rechazo }\end{array}$ & Rechazo & Indiferencia & Aceptación & 75 \\
\hline \multirow{2}{*}{ Inicial y Especial } & 1 & 26 & 43 & 5 & $100,0 \%$ \\
\hline \multirow{2}{*}{ Primaria e Informática } & $1,3 \%$ & $34,7 \%$ & $57,3 \%$ & $6,7 \%$ & 54 \\
& 5 & 22 & 23 & 4 & $100,0 \%$ \\
\hline \multirow{2}{*}{ Matemática y Computación } & $9,3 \%$ & $40,7 \%$ & $42,6 \%$ & 7 & 25 \\
& $12,0 \%$ & $44,0 \%$ & $28,0 \%$ & $16,0 \%$ & $100,0 \%$ \\
\hline \multirow{2}{*}{ Total } & 9 & 59 & 73 & 13 & 154 \\
& $5,8 \%$ & $38,3 \%$ & $47,4 \%$ & $8,4 \%$ & $100,0 \%$ \\
\hline
\end{tabular}

Nota: $\mathrm{X}^{2}=11,713 ; \mathrm{p}=0,069>0,05$

Fuente: Base de datos.

La información de la tabla 3 revela que en los estudiantes de la especialidad de Matemática y Computación predomina la actitud de rechazo frente a la educación virtual (44\%) en comparación a los estudiantes de las especialidades de Inicial y Especial $(57,3 \%)$ y Primaria e Informática $\quad(42,6 \%)$, quienes se caracterizan por tener actitudes de indiferencia. Asimismo, se observa que no existen diferencias estadísticamente significativas entre la actitud frente a la educación virtual y la especialidad a la que pertenecen los estudiantes $(\mathrm{p}=0,069>0,05)$. 
Tabla 4 - Actitud de los estudiantes frente a la educación virtual según sexo.

\begin{tabular}{|c|c|c|c|c|c|}
\hline \multirow{2}{*}{ Sexo } & \multicolumn{4}{|c|}{ Actitud frente a la educación virtual } & \multirow{2}{*}{ Total } \\
\hline & Mucho rechazo & Rechazo & Indiferencia & Aceptación & \\
\hline \multirow{2}{*}{ Masculino } & 2 & 10 & 28 & 7 & 47 \\
\hline & $4,3 \%$ & $21,3 \%$ & $59,6 \%$ & $14,9 \%$ & $100,0 \%$ \\
\hline \multirow{2}{*}{ Femenino } & 7 & 49 & 45 & 6 & 107 \\
\hline & $6,5 \%$ & $45,8 \%$ & $42,1 \%$ & $5,6 \%$ & $100,0 \%$ \\
\hline \multirow{2}{*}{ Total } & 9 & 59 & 73 & 13 & 154 \\
\hline & $5,8 \%$ & $38,3 \%$ & $47,4 \%$ & $8,4 \%$ & $100,0 \%$ \\
\hline
\end{tabular}

Nota: $X^{2}=10,866 ; p=0,012<0,05$

Fuente: Base de datos.

Los datos de la tabla 4 indican que los varones presentan actitudes ligeramente más favorables que las mujeres frente a la educación virtual, ya que se caracterizan por tener actitudes de indiferencia $(59,6 \%)$ mientras que en ellas predomina la actitud de rechazo $(45,8 \%)$. Estas diferencias de sexo resultaron ser estadísticamente significativas $(\mathrm{p}=0,012<0,05)$.

Tabla 5 - Actitud de los estudiantes frente a la educación virtual según grupos de edad.

\begin{tabular}{|c|c|c|c|c|c|}
\hline \multirow{2}{*}{ Grupos de edad } & \multicolumn{4}{|c|}{ Actitud frente a la educación virtual } & \multirow{2}{*}{ Total } \\
\hline & Mucho rechazo & Rechazo & Indiferencia & Aceptación & \\
\hline \multirow{2}{*}{ De 16 a 25 años } & 7 & 47 & 66 & 9 & 129 \\
\hline & $5,4 \%$ & $36,4 \%$ & $51,2 \%$ & $7,0 \%$ & $100,0 \%$ \\
\hline \multirow{2}{*}{ De 26 a 35 años } & 0 & 11 & 7 & 3 & 21 \\
\hline & $0,0 \%$ & $52,4 \%$ & $33,3 \%$ & $14,3 \%$ & $100,0 \%$ \\
\hline \multirow{2}{*}{ De 36 a 45 años } & 2 & 1 & 0 & 1 & 4 \\
\hline & $50,0 \%$ & $25,0 \%$ & $0,0 \%$ & $25,0 \%$ & $100,0 \%$ \\
\hline \multirow{2}{*}{ Total } & 9 & 59 & 73 & 13 & 154 \\
\hline & $5,8 \%$ & $38,3 \%$ & $47,4 \%$ & $8,4 \%$ & $100,0 \%$ \\
\hline
\end{tabular}

Nota: $X^{2}=21,634 ; p=0,001<0,05$

Fuente: Base de datos.

En la tabla 5 se observa que los estudiantes cuyas edades oscilan entre los 16 y 25 años se caracterizan por tener una actitud de indiferencia frente a la educación virtual, sin embargo, en el caso del grupo de estudiantes que tienen entre 26 y 35 años $(52,4 \%)$ así como los estudiantes que tienen entre 36 y 45 años (50\%), predomina una actitud de rechazo y mucho rechazo respectivamente. Dichas diferencias fueron estadísticamente significativas $(\mathrm{p}=0,001<0,05)$.

\section{Discusión}

La educación virtual es una modalidad educativa bastante interesante e importante que se ha masificado globalmente en los últimos años debido especialmente al interés de las personas que tienen la intención de continuar estudiando a pesar de tener responsabilidades laborales o familiares. En el caso de las universidades peruanas y en general, de todo el mundo, tuvieron que 
adoptar la educación virtual debido a las disposiciones de aislamiento social obligatorio dadas por los diferentes gobiernos a causa de la creciente tasa de contagios producidos por la pandemia de COVID-19, la cual no permitió que realicen una planificación correcta y no se prevean las limitaciones en cuanto a la carencia de recursos tecnológicos y de acceso a internet, factores determinantes en el éxito de esta modalidad.

En función a lo expuesto, la presente investigación se centró en analizar las actitudes de los estudiantes de la Universidad Nacional Amazónica de Madre de Dios frente a la educación virtual. En ese orden de ideas, un primer resultado indica que los estudiantes se caracterizan por presentar actitudes de indiferencia frente a esta modalidad de enseñanza - aprendizaje, lo cual significa que a pesar de conocer sus beneficios $\mathrm{y}$ ventajas, no se sienten a gusto de desenvolverse virtualmente ni tienen una actitud favorable para involucrarse en el desarrollo de las actividades propuestas. Esto sería explicado por las inadecuadas estrategias pedagógicas de algunos docentes, quienes no están capacitados para enseñar de manera virtual ni conocen herramientas digitales para optimizar el logro de aprendizajes. Asimismo, otro factor para que los estudiantes no tengan actitudes de aceptación sería la carencia de recursos tecnológicos apropiados para desenvolverse e interactuar bajo esta modalidad (laptops, computadoras, tablets y smartphones) así como el limitado acceso al internet de alta velocidad, lo cual genera en ellos a su vez ansiedad y estrés.

Este resultado coincide parcialmente con los hallazgos de Chávez y Morales (2018) quienes investigaron sobre las actitudes de los estudiantes universitarios de psicología ante la educación en línea, concluyendo que presentaban actitudes poco favorables para desenvolverse bajo esta modalidad, es decir, a pesar de que muchos de ellos pertenecían a la generación de nativos digitales, no implicaba que pudieran tener un interés y tendencia positiva para formarse bajo la modalidad virtual.

No obstante, el resultado de la presente investigación expuesto líneas arriba difiere de los hallazgos reportados por Hernández, Fernández y Pulido (2018), Pulido (2017) y Maldonado y Vega (2015) quienes investigaron acerca de las actitudes de los estudiantes guatemaltecos y españoles de pregrado y posgrado ante la educación virtual, concluyendo que la mayoría de ellos presentan una actitud favorable hacia la educación virtual y a sus herramientas digitales. 
Estas diferencias en los resultados se deberían a que en las universidades guatemaltecas y españolas donde se realizaron ambas investigaciones la modalidad de enseñanza virtual se había implementado previamente y contaban con el recurso humano capacitado y los recursos virtuales adecuados para llevarse a cabo. En cambio, la Universidad Nacional Amazónica de Madre de Dios implementó la educación virtual de manera improvisada debido a las repentinas disposiciones de aislamiento social obligatorio causado por el COVID-19, no teniendo oportunidad de fortalecer las capacidades tecnológicas y didácticas de los docentes, no contar con el personal de soporte técnico ni prever la gestión de recursos económicos para apoyar a los estudiantes con limitaciones económicas que no tenían los equipos tecnológicos ni el acceso a internet para ser parte de la modalidad de enseñanza - aprendizaje virtual.

Otro hallazgo importante en la presente investigación indica que no se encontraron diferencias significativas entre la actitud frente a la educación virtual y la especialidad a la que pertenecen los estudiantes, no obstante, los estudiantes de las especialidades de Inicial y Especial y Primaria e Informática presentaron actitudes ligeramente más favorables que los estudiantes de la especialidad de Matemática y Computación. A pesar que dos especialidades están bastante vinculadas al dominio de las tecnologías de la información y comunicación (Primaria e Informática y Matemática y Computación), llama la atención que esta última presenta actitudes menos favorables hacia la educación virtual. En ese sentido, lo expuesto contradeciría lo hallado y expresado por Hernández, Fernández y Pulido (2018), Pilli, Fanaeian y AlMomani (2014) y Waern y Ramberg (1996) quienes señalaron que el conocimiento previo o la experiencia computacional influye en la percepción de los estudiantes sobre el aprendizaje en entornos virtuales.

Del mismo modo, también se encontró diferencias estadísticamente significativas entre la actitud frente a la educación virtual y el sexo de los estudiantes, es decir, los varones presentaron actitudes ligeramente más favorables que las mujeres. Este hallazgo concuerda con los resultados de diversas investigaciones (Martínez, 2012; Chávez y Morales, 2018 y Marshall y Ruiz, 1999) quienes encontraron que los estudiantes universitarios varones presentaban mayores puntuaciones y por ende, niveles de predisposición y aceptación a la 
educación en línea así como sus herramientas digitales.

Por otra parte, se hallaron diferencias estadísticamente significativas entre la actitud frente a la educación virtual y el grupo de edad de los estudiantes, es decir, los estudiantes más jóvenes (de 16 a 25 años) presentaron actitudes de indiferencia mientras que en los estudiantes cuyas edades eran superiores (de 26 a 45 años) predominaba una actitud de rechazo y mucho rechazo. Estos resultados serían explicados por el mayor dominio y comprensión de los estudiantes de los entornos virtuales de aprendizaje, ya que en su formación previa (educación secundaria) cursaron asignaturas de computación y tienden a adaptarse mejor a la utilización de herramientas tecnológicas en comparación a los estudiantes de mayor edad, a quienes posiblemente les cuesta adaptarse a la modalidad virtual y al uso de sus múltiples recursos de aprendizaje.

Los hallazgos expuestos líneas arriba divergen de las investigaciones de Martínez (2012), Hernández, Fernández y Pulido (2018) en las cuales se encontraron también diferencias significativas, sin embargo, demostraron que las personas de edades superiores tenían mayor disposición y actitud a desarrollar las actitudes propuestas bajo la modalidad virtual en comparación a los estudiantes jóvenes.
Esto ocurriría ya que muchos de los estudiantes cuyos grupos de edades eran superiores tenían responsabilidades laborales y en sus hogares por lo que la modalidad virtual se presentaba como una estrategia idónea para continuar sus estudios y culminarlos.

\section{Consideraciones finales}

En la actualidad la educación virtual permite la formación académica de los estudiantes a través de las TIC, optimizando de esta manera el proceso de enseñanza - aprendizaje. Bajo esa perspectiva, debe fortalecerse y masificarse a nivel regional, nacional e internacional debido a la accesibilidad, flexibilidad y facilidad de uso, características importantes que permiten el mayor acceso a la educación superior así como la permanencia y la reducción de los índices de deserción estudiantil. Sin embargo, su implementación también supone un complejo proceso de planificación que promueva el fortalecimiento de las competencias digitales de los docentes, la adopción de un aula virtual amigable, con herramientas y recursos digitales que fomenten el logro de competencias y capacidades en los estudiantes, el soporte técnico necesario y garantizar la conectividad a internet de alta velocidad, 
especialmente a los estudiantes con limitaciones económicas.

Lo expuesto concuerda con los planteamientos de Hernández, Fernández y Pulido (2018), quienes señalan que cuando una institución implementa el aprendizaje virtual de calidad, de fácil acceso y uso así como flexible, mejora los niveles de motivación y disposición de sus estudiantes para aprovechar y beneficiarse de dicha oferta de aprendizaje. Esto supone además que las actitudes que tengan los estudiantes frente a la educación virtual podrían ser factores a tomarse en cuenta para su diseño, implementación y desarrollo.

A partir de los resultados hallados en la presente investigación se pudo concluir que la actitud que presentaron los estudiantes frente a la educación virtual es de indiferencia. Con relación a las dimensiones, se identificó que los estudiantes también muestran indiferencia ante percepción de utilidad, la intención de adoptar la modalidad virtual así como la facilidad de uso. Por otro lado, evidencian una actitud de rechazo ante el soporte pedagógico y técnico y sus niveles de estrés y ansiedad al desenvolverse virtualmente incrementaron, en comparación a la modalidad presencial. Finalmente, se encontraron diferencias estadísticamente significativas entre la actitud frente a la educación virtual y el sexo así como la edad, mas no con la especialidad a la que pertenecían los estudiantes.

Es necesario precisar que las conclusiones expuestas se limitan al grupo de estudiantes universitarios que formaron parte de la investigación por lo que se recomienda que futuras investigaciones incrementen la muestra de estudio, considerando a estudiantes de otras carreras profesionales para poder confirmar los resultados y ampliar su generalización. Del mismo modo, los instrumentos de recolección de datos, al haber sido autoadministrados, podrían generar un sesgo de deseabilidad social en los resultados, por lo que se sugiere aplicar otros instrumentos que complementen $\mathrm{y}$ garanticen su objetividad. Finalmente, se recomienda que los futuros estudios incluyan las percepciones de los docentes para que los hallazgos demuestren de manera integral cómo se viene dando la educación virtual y sirva como antecedente para realizar los reajustes y medidas correctivas en aras de brindar un servicio educativo universitario de calidad.

\section{Agradecimiento}

Expresamos nuestro más profundo agradecimiento a los docentes y estudiantes de la carrera profesional de 
Educación de la Universidad Nacional Amazónica de Madre de Dios que gentilmente fomentaron la participación del estudiantado y respondieron las encuestas respectivamente.

\section{Referencias}

Aguilar, D. (2015). Ser docente virtual: tiempo y presencia (Tesis de Posgrado). Universidad de Málaga, Málaga, España. Recuperado de http://hdl.handle.net/10630/12380

Ajzen, I. (1991). The theory of planned behavior. Organizational behavior and human decision processes, 50(2), 179-211. https://doi.org/10.1016/0749-

\section{8(91)90020-T}

Campillo, C., Morales, N., Trejo, H., Ramírez, J., Castañeda, I., Gallegos, R., Gálvez, A., y Rosas, C. (2013). La educación en línea: una metodología flexible para formación de residentes de Psiquiatría. Investigación en Educación Médica, 2(6), 87-93. https://doi.org/10.1016/S20075057(13)72692-9

Chávez, J., y Morales, M. (2018). Actitud hacia la educación en línea en estudiantes de Psicología. Revista Electrónica del Desarrollo Humano para la Innovación Social, 5(10), 1-13. Recuperado de http://www.cdhis.org.mx/index.php/CAGI/ article/view/129

Cruz, M., y Rama, C. (2016). La educación a distancia y virtual en Centroamérica y El Caribe. Santo Domingo: Ediciones UAPA. Recuperado de https://virtualeduca.org/documentos/observ atorio/2016/la-educacion-a-distancia-encentroamerica-y-el-caribe.pdf
De las Salas, M., Perozo, S., y Lugo, Z. (2015). Actitud del estudiante universitario hacia la investigación en el núcleo luz costa oriental del lago. Revista Electrónica de Humanidades, Educación y Comunicación Social, 18(9), 162-176. Recuperado de http://ojs.urbe.edu/index.php/redhecs/articl e/view/2446

Durán, N. (2015). Caracterización de las prácticas evaluativas en el programa virtual de psicología de la Universidad Nacional Abierta y a Distancia. (Tesis de Posgrado). Universidad Pedagógica Nacional, Colombia. Recuperado de http://hdl.handle.net/20.500.12209/917

Durán, R. (2015). La educación virtual universitaria como medio para mejorar las competencias genéricas y los aprendizajes a través de buenas prácticas docentes. (Tesis de Posgrado). Universidad Politécnica de Cataluña, España. Recuperado de http://hdl.handle.net/10803/397710

Durán, R., Estay, C., y Álvarez, H. (2015). Adopción de buenas prácticas en la educación virtual en la educación superior. Aula Abierta, 43(2), 77-86. https://doi.org/10.1016/j.aula.2015.01.001

Gómez, L., y Macedo, J. (2011). Importancia de los programas virtuales en la educación superior peruana. Investigación Educativa, 15(27), 113-126. Recuperado de https://revistasinvestigacion.unmsm.edu.pe /index.php/educa/article/view/5169

Hernández, R. Fernández, C., y Baptista, P. (2014). Metodología de la Investigación. (6 $6^{\mathrm{a}}$ d.). México: Mc Graw-Hill

Hernández, V., Fernández, K., y Pulido, J. (2018). La actitud hacia la educación en línea en estudiantes universitarios. Revista De Investigación Educativa, 36(2), 349- 
364.

https://doi.org/10.6018/rie.36.2.277451

Herrera, C., Espinoza, M., Ludeña., V., y Michay, G. (2019). Las Tics como herramienta de interacción y colaboración en el área de Biología. Revista Espacios, 40(41), 1-10. Recuperado de https://www.revistaespacios.com/a19v40n $\underline{\text { 41/19404101.html }}$

Instituto de Investigaciones sobre la Universidad y la Educación (2020). Educación y pandemia. Una visión académica. México: UNAM. Recuperado de

http://www.iisue.unam.mx/nosotros/covid/ educacion-y-pandemia

Instituto Nacional de Estadística e Informática (2018) Perú Perfil sociodemográfico Informe Nacional Censos nacionales 2017: XII de población VII de vivienda y III de comunidades indígenas. Lima: INEI.

Lara, L. (2002). Análisis de los recursos interactivos en las aulas virtuales. Ponencia presentada en el Segundo Congreso Virtual Integración sin barreras en el siglo XXI, Argentina.

Maldonado, G., y Vega, E. (2015). Actitud de los estudiantes universitarios ante la plataforma Moodle. Píxel-Bit. Revista de Medios y Educación, 47, 105-117. https://doi.org/10.12795/pixelbit.2015.i47. $\underline{07}$

Marshall, B., y Ruíz, E. (1999). Las actitudes de los-as alumnos-as de enseñanza secundaria hacia los ordenadores en función del género. En EDUTEC 99. IV Congreso de Nuevas Tecnologías de la Información y de la Comunicación para la educación. Nuevas tecnologías en la formación flexible y a distancia. Sevilla, España. Recuperado de http://hdl.handle.net/11441/63000
Martínez, P. (2012). Actitud hacia la educación en línea según el estilo predominante de aprendizaje, el género y el nivel de estudio. En V Congreso Mundial de Estilos de Aprendizaje. Congreso llevado a cabo en Santander, España. Recuperado de https://dialnet.unirioja.es/servlet/articulo?c $\underline{\text { odigo }=4640481}$

Mehra, V., y Omidian, F. (2012). Development an instrument to measure university student's attitude towards elearning. Turkish Online Journal of Distance Education, 13(1), 34-51. Recuperado de http://tojde.anadolu.edu.tr/makale_goster.p $\underline{\mathrm{hp} ? \mathrm{id}=728}$

Moreira, C., y Delgadillo, B. (2015). La virtualidad en los procesos educativos: reflexiones teóricas sobre su implementación. Tecnología en Marcha, 28(1), 121-129. Recuperado de http://www.scielo.sa.cr/scielo.php?script=s ci arttext\&pid=S0379-

$\underline{39822015000100121 \& \operatorname{lng}=\mathrm{en} \& \operatorname{tlng}=\mathrm{es}}$

Moreno, S. (20209. La innovación educativa en los tiempos del Coronavirus. Salutem Scientia Spiritus, 6(1), 14-26. Recuperado de https://revistas.javerianacali.edu.co/index.p $\mathrm{hp} /$ salutemscientiaspiritus/article/view/229 $\underline{0}$

Núñez, J. (2020). COVID-19 por SARSCov2 también ha afectado a la Educación Médica. Educación Médica, avance de publicación en línea.

Pando, V. (2018). Tendencias didácticas de la educación virtual: Un enfoque interpretativo. Propósitos y Representaciones, 6(1), 463-505. https://doi.org/10.20511/pyr2018.v6n1.167

Patiño, A., y Palomino, L. (2004). Fundamentos pedagógicos. Lima: Pontificia Universidad Católica del Perú. 
Pilli, O., Fanaeian, Y., y Al-Momani, M (2014). Investigating the Students' Attitude Toward the use of E-Learning in Girne American University. International Journal of Business and Social Science, 5(5), 169-175. Recuperado de https://ijbssnet.com/journal/index/2481

Pulido, J. (2017). Actitud hacia la educación virtual de los alumnos de postgrado de la UPEL. Razón y Palabra, 21(3_98), 606-623. Recuperado de http://www.revistarazonypalabra.org/index .php/ryp/article/view/890

Rivera, A., Viera, L., y Pulgarón, R. (2010). La educación virtual, una visión para su implementación en la carrera de Tecnología de la Salud de Pinar del Río. Educación Médica Superior, 24(2) Recuperado de http://scielo.sld.cu/scielo.php?script=sci_ar ttext\&pid=S0864-

$\underline{21412010000200002 \& \operatorname{lng}=\text { es\&tlng=es }}$

Sánchez, H., Reyes, C., y Mejía, K. (2018). Manual de términos en investigación científica, tecnológica y humanística. Lima: Universidad Ricardo Palma. Recuperado de http://repositorio.urp.edu.pe/handle/URP/1 $\underline{480}$

Waern, Y., y Ramberg, R. (1996). People's perception of human and computer advice. Computers in Human Behavior, 12(1), 17$27 . \quad$ https://doi.org/10.1016/0747$\underline{5632(95) 00016-X}$

\begin{abstract}
Información del articulo / Article Information
Recibido en: 04/08/2020

Aprobado en: $17 / 08 / 2020$

Publicado en: 08/09/2020

Received on August 04th, 2020

Accepted on August 17th, 2020

Published on September, 08th, 2020

Contribuciones en el artículo: Los autores fueron responsables de todas las etapas y resultados de la investigación, a saber: elaboración, análisis e interpretación de los datos; redacción y revisión del contenido del manuscrito y; aprobación de la versión final publicada.
\end{abstract}

Author Contributions: The author were responsible for the designing, delineating, analyzing and interpreting the data, production of the manuscript, critical revision of the content and approval of the final version published.

Conflictos de Intereses: Los autores han declarado que no existe conflicto de intereses con respecto a este artículo.

Conflict of Interest: None reported.

Orcid

Edwin Gustavo Estrada Araoz

iD http://orcid.org/0000-0003-4159-934X

Néstor Antonio Gallegos Ramos

(iD) http://orcid.org/0000-0003-1436-9207

Helen Juddy Mamani Uchasara

iD http://orcid.org/0000-0003-1735-8570

Karl Herbert Huaypar Loayza

iD http://orcid.org/0000-0002-4809-7134

Cómo citar este artículo / How to cite this article

APA

Estrada-Araoz, E. G., Gallegos-Ramos, N. A., MamaniUchasara, H. J., \& Huaypar-Loayza, K. H. (2020). Actitud de los estudiantes universitarios frente a la educación virtual en tiempos de la pandemia de COVID-19. Rev. Bras. Educ. Camp., 5, e10237. http://dx.doi.org/10.20873/uft.rbec.e10237

ABNT

ESTRADA-ARAOZ, E. G.; GALLEGOS-RAMOS, N. A.; MAMANI-UCHASARA, H. J.; \& HUAYPAR-LOAYZA, K. H. Actitud de los estudiantes universitarios frente a la educación virtual en tiempos de la pandemia de COVID19. Rev. Bras. Educ. Camp., Tocantinópolis, v. 5, e10237, 2020. http://dx.doi.org/10.20873/uft.rbec.e10237 